\title{
Should Hand-Assisted Laparoscopic Surgery Be Placed in the Realm of Minimally Invasive Surgery?
}

\author{
Hungdai Kim \\ Department of Surgery, Kangbuk Samsung Medical Center, Sungkyunkwan University School of Medicine, Seoul, Korea
}

See Article on Page 72-76

Since its first report in 1991, conventional laparoscopic surgery has become a standard procedure for a colon resection worldwide [1]. Various novel techniques, such as Notes, single-port laparoscopic surgery, and robot-assisted laparoscopic surgery, have been are introduced and the scopes of their activities have been increasing.

Currently, hand-assisted laparoscopic surgery (HALS) is employed in three scenarios. First, HALS is accepted as an alternative to conventional laparoscopic surgery. Second, HALS is used for a transient period of training as a stepping-stone to conventional laparoscopic surgery proficiency. Lastly, some surgeons specifically use this technique for more complex cases.

HALS is not a popular technique in Korea. Most laparoscopic surgeons in Korea believe that HALS is an unnecessary procedure or is not different from open surgery. Because HALS requires the surgeon's hand to be put in the patient's abdominal cavity, this type of operation is thought to violate the minimally invasive concept of conventional laparoscopic surgery. Thus, most Korean surgeons use HALS in the 2nd and 3rd scenarios described above. However, surgeons who prefer HALS assert that this procedure has the advantages of both open and conventional laparoscopic surgery. A report by Yang et al. [2], as well as many other reports in the literature, agree that HALS retains the short-term benefits of conventional laparoscopic surgery, such as smaller incision, lesser pain, faster recovery, and shorter hospital stay. Besides, if the surgeon who seldom perform HALS, it is hard to obtain its

Correspondence to: Hungdai Kim, M.D.

Department of Surgery, Kangbuk Samsung Medical Center, Sungkyunkwan University School of Medicine, 29 Saemunan-ro, Jongno-gu,

Seoul 110-746, Korea

Tel: +82-2-2001-2137, Fax: +82-2-2001-2131

E-mail:hungdai.kim@samsung.com

(c) 2013 The Korean Society of Coloproctology

This is an open-access article distributed under the terms of the Creative Commons Attribution NonCommercial License (http://creativecommons.org/licenses/by-nc/3.0) which permits unrestricted noncommercial use, distribution, and reproduction in any medium, provided the original work is properly cited. own advantages [2-4].

The authors compared a HALS right hemicolectomy with an open right hemicolectomy. In common with many other reports in the literature, they found the HALS technique to be equal to or better than open surgery with respect to postoperative clinical parameters $[5,6]$. Among the several advantages of HALS, the authors particularly emphasized restoration of tactile feedback to surgeon's hand during the procedure. This point is very different from conventional laparoscopic surgery and contributes greatly to the surgery being easier and shorter. In my own view, this point also supports surgeon's emotional stability by increasing self-confidence. Because the surgeon's hand is in patient's abdomen, handling unexpected situations that may happen during surgery is much easier and faster than it is with conventional laparoscopic surgery.

Conventional laparoscopic surgery is still criticized because of its being a demanding technique, lacking tactile sensation, prolonging procedural time and having a steep learning curve. I am certain that as long as these drawbacks of the in conventional technique exist, HALS will continue to be an option for surgeons. To support the superiority of the HALS technique, the authors should analyze long-term survival data in the future. Furthermore, in the current minimally invasive surgery era, a comparison between HALS and conventional laparoscopic surgery is essential for correct positioning.

In conclusion, I believe that the hand of an experienced surgeon is the best instrument in the operating room. Technical improvement and development of high-end instruments for HALS is needed if it is to gain wide popularity among surgeons.

\section{REFERENCES}

1. Jacobs M, Verdeja JC, Goldstein HS. Minimally invasive colon resection (laparoscopic colectomy). Surg Laparosc Endosc 1991;1: 144-50.

2. Yang I, Boushey RP, Marcello PW. Hand-assisted laparoscopic colorectal surgery. Tech Coloproctol 2013;17 Suppl 1:S23-7.

3. Ringley C, Lee YK, Iqbal A, Bocharev V, Sasson A, McBride CL, et al. Comparison of conventional laparoscopic and hand-assisted 
oncologic segmental colonic resection. Surg Endosc 2007;21:213741.

4. Vogel JD, Lian L, Kalady MF, de Campos-Lobato LF, Alves-Ferreira PC, Remzi FH. Hand-assisted laparoscopic right colectomy: how does it compare to conventional laparoscopy? J Am Coll Surg 2011;212:367-72.

5. Sim JH, Jung EJ, Ryu CG, Paik JH, Kim G, Kim SR, et al. Short- term outcomes of hand-assisted laparoscopic surgery vs. open surgery on right colon cancer: a case-controlled study. Ann Coloproctol 2013;29:72-6

6. Chung CC, Ng DC, Tsang WW, Tang WL, Yau KK, Cheung HY, et al. Hand-assisted laparoscopic versus open right colectomy: a randomized controlled trial. Ann Surg 2007;246:728-33. 